\title{
OPTICAL TRANSMITTANCE OF FUSED SILICA AT ELEVATED TEMPERATURES DURING HIGH ENERGY ELECTRON BOMBARDMENT
}

by Albert B. Smith

Lewis Research Center

Cleveland, Ohio

TECHNICAL PAPER proposed for presentation at

Uranium Plasma Conference sponsored by the American

Institute of Aeronautics and Astronautics

Atlanta, Georgia, November 15-17, 1971 
OFIICAL TRANSMITTANCE OF FUSED SПICA AT ELEVATED TEMPERATURES DURING HTGH ENERGY ELECTRON BOMBARDMENT

Albert B. Smith

Lewis Research Center

National Aeronautics and Space Administration

Cleveland, Ohio

\section{Abstract}

An experimental determination of the optical transmission of Corming $7940 \mathrm{UV}$ and Suprasil I fused silica has been made. The LeRC dynamitron provided the equivalent ionizing radiation and high temperature that the transparent gas divider of an operating nuclear light bulb engine would experience. The irradiation induced absorption was measured at $2150 \AA, 2700 \AA$, and $4500 \AA$. The length of the irradiations were sufficient so that an equilibrium between radiation induced coloration and high temperature annealing was reached. The experimental results indicate a significant optical absorption, particularly at the shorter wavelength, which would make the use of fused silica in this concept questionable.

\section{Introduction}

One interesting nuclear propulsion concept is the gaseous core nuclear reactor. In one design fused silica is proposed as a transparent wall separating the fuel and the propellant. This Nuclear Iight Bulb transfers the energy of the fissioning Iuel by thermal radiation through the fused silica to a hydrogen propellant seeded with opaque particles. The radiant energy obsorbed lies in the range of $2000 \AA$ to $10000 \AA$.

Practical realization of this propulsion scheme depends in large measure upon the transparency of these cooled fused silica walls at these wavelengths, since the energy must radiate through them. The objective of the present work is to measure under simulated reactor environment, at the wavelengths of interest, the optical transmission of fused silica.

During reactor operation, the fused silica will be exposed to a large flux of fast neutrons and gamma rays emanating from the fissioning fuel it surrounds. Therefore, color centers on optically absorbing centers can be generated as a result of the ionization and damage in the wall material; with subsequent transmission loss. The center of the most prominent irradiation-induced absorption band in the fused silica occurs at a wavelength of $2150 \AA$. A subsidiary peak occurs at 2700 . Additional measurements were made at 4500 \& to determine the width of the absorption band and to check absorption where strong absorption bands are not expected to exist.

These radiation-induced absorption centers are stable at room temperatures, but at elevated temperatures they may be effectively annihilated, annealed or bleached. In this experimental work, and in the application of the nuclear rocket the fused silica will be at temperatures of $600^{\circ} \mathrm{C}$ to $1000^{\circ} \mathrm{C}$. So we have two competing processes affecting the transmission: first, coloration induced by nuclear radiation; and second, annealing of coloration by exposure to elevated temperature. This equilibrium transmission must be sufficiently high for the concept to be workable, and its meas- urement is the prime objective of this experiment.

The Lewis Research Center Dynamitron is used to give an electron beam in which the ionizing dose rate is equivalent to an operating full-scale NUB engine. This accelerator provided $1.5 \mathrm{MeV}$ electrons at current densities in the range of 20 to $150 \mu \mathrm{A} / \mathrm{cm}^{2}$. This corresponds to an ionizing dose rate of 2.0 to $10 \mathrm{Mrad} / \mathrm{sec}$ deposited in the specimen, which exceeds anything available in any existing test reactor known today. It was also possible to run for times comparable to the $1000 \mathrm{sec}$ duration of the full scale engine.

Specimen temperature was governed by electron beam heating; most irradiations were done at 80 to $150 \mu \mathrm{A} / \mathrm{cm}^{2}$ current densities to be at the temperature range of interest $\left(600^{\circ}\right.$ to $\left.900^{\circ} \mathrm{C}\right)$.

A dual beam ratio recording spectrophotometer was designed and used for the measurement of the optical transmission. Samples of Corning Grade $7940 \mathrm{UV}$, and Suprasil (Amersil Inc.) were tested. These samples of fused silica were chosen because of their high purity, structural integrity, resistance to radiation damage, transparency over a wide range of wavelengths and thermal properties.

\section{Experimental Arrangement}

\section{Lewis Dynamitron Electron Accelerator}

The LeRC Dynamitron Electron Accelerator is a potential drop accelerator capable of supplying accelerating potentials between 0.3 to $3.0 \mathrm{MeV}$ with voltage stability greater than 99.9 percent. I The accelerator can provide direct current, monoenergetic electrons or positive ion beams controllable from I $\mu \mathrm{A}$ to $3 \mathrm{~mA}$. In the electron mode of operation a hot tungsten filament is used as a source of particles.

The beam transport system consists of an analyzing magnet, positioning coils and a three element quadrupole focussing set. All power supplies for this system have sufficient stability so that the beam position changes at the output ports are less than 0.1 percent for a monoenergetic beam.

The alignment and focussing of the beam could be monitored by a beam profile monitor and also a closed circuit television system.

\section{Xenon Lamp Source and Supply}

A $1000 \mathrm{~W}$ Xenon arc lamp was used as the primary light source. The Xenon are was maintained by a power supply which was continuously variable and monitored to insure lamp performance. This power supply provided dc regulation to \pm 1 percent with \pm 10 percent change in line voltage. A fast acting control circuit compensates for changes within the lamp and produces maximum arc stability. The current ripple was 1 percent rms. 
Dual Beam Ratio Recording Spectrophotometer

A large dual beam ratio recording spectrometer was designed and built to measure the transmission through the fused silica samples. This system employed two optical beams, a sample beam and a reference beam. The two beams obtained from a single light source, are separated by using a rotating mirror beam chopper which alternately passes light to the sample mirror or the reference path. Thus the two paths are parallel and separated by approximately $45.72 \mathrm{~cm}$. The beams are collimated to a diameter of $0.72 \mathrm{~cm}$. The sample and the reference light beams are directed at right angles through the faces of the test and reference specimens respectively. The two beam paths are equal in length. They are combined by a serrated surface mirror at the entrance slit of the monochromator. A block diagram of the optical setup is pictured in Fig. 1. The rotating mirror beam chopper sequentially modulates the sample and reference beams so that locked-in signal processing techniques and phase sensitive detection is possible. The modulating frequency is $13 \mathrm{~Hz}$, and the reference voltage obtained from the rotating mirror triggers the lock in amplifier. Only the modulation component of the detection signal is rectified to produce a dc output from the instrument. In this way, we discriminated against the noise sources such as sample fluorescence, ambient room light, Xenon lamp fluctuations or any remaining Bremsstrahling radiation, and are able to read an extremely weak signal while at the same time ignoring this large background of noise.

The monochromator could select a particular wavelength range is between 160 and 3500 millimicrons. Since both sample and reference beams have common detection and amplifier components and ratio recording (or the comparison of sample beam intensity with reference beam intensity was used) inaccuracies due to amplifier gain or spectral response of the photomultiplier is eliminated.

\section{Optical Base}

The Xenon Iamp, monochromator, mirrors and beam splitters, reference path furnace and specimen holder box were all securely fastened to a (91.4 cm wide) $\times(182.9 \mathrm{~cm}$ long $) \times(3.82 \mathrm{~cm}$ deep $)$ aluminum table with a top surfaced machined flat to $0.050 \mathrm{~mm}$. Since 2.11 these parts are held in their relative positions, the whole base could be moved without disturbing the critical beam alignment. The complete system was then suspended from the ceiling by unistruts to completely isolate it from any vibrations caused by the vacuum pumps on the electron-beam transport system.

\section{Electronic System}

The control panel containing the phasesensitive amplifier and the remaining electronic equipment was located in the dymanitron control room located out of the irradiation area and separated by $30.5 \mathrm{~m}$. The detection system included a 4 pen chart recorder which simultaneously recorded furnace temperature, specimen temperature, specimen holder temperature, and the amplified ratio between specimen and reference beams. Readout of the wavelengths and actual position of the electron beam on the specimen was accomplished by closed circuit television camera remotely con- trolled from the dynamitron control console. These were monitored continuously while the test was being run. The output of the phase-sensitive amplifier was checked by viewing the signal and the rectified signal on a dual oscilloscope.

The photomultiplier tube was shielded by several lead blocks to minimize the noise generated by $\mathrm{X}$-rays present during the irradiations. Since the signal cables were so long low capacity cables were used and a line matching driver amplifier was connected after the pre-amplifier to prevent signal loss.

This system was capable of measurement of optical transmission with an accuracy of \pm 1 percent. Response time was limited by the chart recorders which was approximately 1 sec.

\section{Specimen Configuration}

The specimen configuration for this experiment was a compromise which met the various requirements of the test. It was sized so that the correct electron energy deposited in the specimen would give the necessary ionizing dose rates. The specimen was thick enough to allow for accurate measurement of the optical transmission for absorption coefficient from $0.05 \mathrm{~cm}^{-1}$ to $20 \mathrm{~cm}^{-1}$. The optical path through it was normal to its surface to minimize the effects of thermally induced refractive index gradients, and possible temperature induced stresses which might lead to erroneous transmission determination. The specimens were of wafer form $2.54 \mathrm{~cm}$ square and $1.5 \mathrm{~mm}$ thick. They were polished flat to $1 / 4$ wavelength of Sodium light and parallel to 2 wavelengths. 'the specimen was at right angles with respect to the optical beam axis and $20^{\circ}$ with respect to the electron beam axis.

\section{Specimen Holder}

The specimen is held in a metal picture frame type clamp. Figure 2 shows the specimen with the chromel-alumel thermocouple embedded in it and the thermocouple spot-welded to the holder. The thermocouple was wedged tight at the center of the sample by using a tapered hole through the fused silica. Spring tension of its leads and thermo expansion of the thermocouple ball tended to secure its position throughout the test. The frame type clamp allowed the sample to be held rigidly or a ceramic gasket material could be inserted between the metal holder and the fused quartz for stress free mounting. The holder also provided cooling slits which blew a sheet of Helium across the faces of the specimen. At the same time cooling gas was directed against the aluminum foil through which the electrons passed. The foil holder was also cooled by water jackets and the gas cooling could be omitted if desired. This double cooling method could be used when very high machine currents were used. Each cooling line was valved and adjustable. The whole specimen holder assembly, which included the cooling tubes, thermocouples, and thermocouple connectors and fittings, was removable from the sample box. It was grounded to the system to help prevent any charge buildup, repeatable positioning of the specimen was guaranteed by eight bolt holes and a centering edge. Readout of the remotely operated upstream beam stop and the thermocouples was con- 
stantly recorded during the experiment.

\section{Sample Box}

The specimen was inserted in the holder and then tine holder bolted to the sample box. The box provided whatever environment was necessary air, helium or vacuum, for the specimen under test. It was connected airectly to the beam transport tube and therefore included the aluminum foil (approximately $0.05 \mathrm{~mm}$ thick). In this way, the Dynamitron vacuum was not affected by the environment used in the test. The sample box also provided collimation for the optical light beam before and after the specimen. It could be sealed by using $\mathrm{SiO}_{2}$ end windows. Permanent magnets were used to prevent the windows from being darkened. It was possible to see the specimen during the experiment by remote television camera through another $\mathrm{SiO} 2$ port directly inline with the $20^{\circ}$ electron beam line.

Therefore, specimen luminescence could be visually observed, and by using a $\mathrm{ZnS}$ coating on the aluminum foil, the beam spot could be continually monitored throughout the experiment. Any beam wandering could be instantly seen. In addition the size and position of the beam spot was determined by a separate test using PVC plastic indicators at the positions of the Al window sample and the Faraday cup. This was done as a standard procedure before each test run.

\section{Calibration}

Calibration of the electron beam striking the specimen was done by removing the fused silica holder and inserting a water-cooled or aircooled Faraday cup. The Faraday cup was positioned exactly the same as the specimen and held by the same eight bolts. It had a guard ring to prevent erroneous contributions from any scattered secondary electrons. In this way external beam current hitting the specimen was calibrated against the internal machine beam current and dial setting.

\section{Absorption Data Analysis}

The 4 channel Bristol recorder simultaneously records specimen temperature $\mathrm{T}_{\mathrm{S}}$, ambient or furnace (reference path) temperature, $\mathrm{T}_{\mathrm{F}}$, holder temperature $\mathrm{T}_{\mathrm{H}} \mathrm{H}$, and the ratio of the specimen to reference signals as a function of elapsed time, $t$. These two signals which constitute the output of the phase sensitive detector, are proportional to the intensity of the sample and reference beams. Thus a record of relative transmission and temperature during the electron irradiation is accomplished. If a single test specimen only was used, the light transmitted I, through its flat, parallel faces is a fraction of the incident light $I_{0}$ given by $I / I_{0}$. This fraction represents the light lost through absorption and reflection if interference effects are ignored. For a specimen of thickness $l$, the fraction lost through absorption is of the form $e^{-\alpha l}$. Multiple reflections in the specimen at the glass-air interface effects the total transmitted intensity. The light transmitted through the first face is $I_{0} e^{-\alpha l}(I-r)$ and $I_{0} e^{-\alpha l}(I-r)^{2}$ through the second face.

The total transmitted intensity may be summed 2,3 and expressed as:

$$
I=I_{0} e^{-a l}(I-r)^{2}\left[I-\left(e^{-a l}\right)^{2} r^{2}\right]^{-1}
$$

or

$$
\frac{I}{I_{0}}=\frac{e^{-a l}(1-r)^{2}}{\left[1-\left(e^{-a l)^{2}} r^{2}\right]\right.}
$$

where $\alpha$ is the absorption coefficient at a particular wavelength, $r$ is the reflection loss, and is related to the index of refraction $n$ by the relation:

$$
r=\left(\frac{n-1}{n+1}\right)^{2}
$$

For fused silica, in the wavelength range we are considering, $n$ does not exceed $1.6^{4}$ and hence the denominator of $\mathrm{Eq}$. (2) is essentially (I) assuming the no absorption case where $e^{-a l}=1$ so that multiple reflections can be safety ignored. So the reflection loss is just $(1-r)^{2}$, and we may write:

$$
\frac{I}{I_{0}}=(1-x)^{2} e^{-a \ell}
$$

or

$$
\alpha=-\frac{1}{\ell}\left[\left(\ln \frac{I}{I_{0}}\right)-\ln (I-r)^{2}\right]
$$

In this experiment a reference specimen was provided of the exact same composition as the test sample, in the axial crusilite vacuum furnace of the reference optical path. This technique effectively cancels reflection losses so that Eq. (5) reduces to simply:

$$
\alpha=-\frac{1}{l} \ln \frac{I(\lambda, \dot{D}, t, T)}{I_{O}(\lambda)}
$$

where $I_{0}(\lambda)$ is the chart recorder amplitude before the electron beam is turned on and $I(\lambda, \dot{D}, t, T)$ the recorder amplitude at a time $t$, after the beam is on.

The raw data from the recorder strip chart of optical transmission, specimen temperature, and electron current density or ionizing dose rate are presented as the electron irradiation history of each specimen by converting the optical transmission to radiation induced absorption coefficient (by use of eq. (6)) and graphing these parameters as a function of elapsed time. Typical raw data is shown in Fig. 3, in this case for a wavelength of $2700 \AA$ and a specimen $1.5 \mathrm{~mm}$ thick of Suprasil I.

A useful computational scheme devised by Burrel, Wright, and Watts ${ }^{5}$ is used to calculate the ionizing dose rate through the specimens. This semi-empirical technique is based on the Monte Carlo electron energy transfer data of Berger. ${ }^{6}$ Using this technique, an approximate result is calculated for the complex relation between the ionizing dose rate, $\dot{D}$, and the electron current density, J, for an electron of kinetic energy, $\mathrm{E}$, of $1.5 \mathrm{MeV}$ impinging upon the specimen configuration used in this experiment. This 
Sestriqua is described in the appendix. the realis are now discussed.

Te tonizing dose rate is D - 0.13 i (lirad/ sec). Because or the complexity of the atomic processes involved in determinin: the ionizin iose rate, an error of \pm 25 percent is estimated. A finl-scale IUB engine operatins at a radiating temperafure of $15000^{\circ} \mathrm{R}$ is expected to five an ionizing dose rate of $5.4 \times 10^{6} \mathrm{R} / \mathrm{sec} .7$ This dose rate is well within the current capability of the Letis Dynamitron Electron Accelerator.

Specimen temperature was by electron beam heatins, and most irradiations were done at 80 to $1.50 \mu \mathrm{A} / \mathrm{cm}^{2}$ current densities. Helium cooling gas was also employed at times so that the specimen tempereture was controllable in the range of interest (600 to $900 \mathrm{C}$ ). Satisfactory temperature measurement using the chromel-alumel thermocouples emvedded in the sample, ano the sample holder, had error less than $\pm 20^{\circ} \mathrm{C}$ based on the inherent accuracy oI measuring equipment and the electron beam hitting the thermocouple. Since the designed spectrophotometer was capable of optical transmission measurement under laboratory conaitions of $\pm I$ percent, this gives a measurement accuracy of about \pm 16 percent at all the induced absorption coefficients of between $1.0 \mathrm{~cm}^{-1}$ and $12 \mathrm{~cm}^{-1}$.

Figure 4 presents the electron irradiation history of a $1.5 \mathrm{~mm}$ Surpasil I specimen at $2150 \AA$. Here the ionizing dose rate is raised in step intervals. It can be seen that both the temperature and the absorption coefficient is increased correspondingly and that the temperature does lie in the range of interest.

There is a slow decrease in this absorption coefficient in time at this temperature. Generally, the induced absorption coefficient is decreased very slightly from its initial value at the outset of the irradiation but does not go to very low values until the bombardment is stopped.

Although $2150 \AA$ is the wavelength of primary interest, the temporal behavior at two other wavelengths was investigated, namely at $2700 \AA$ and $4500 \mathrm{~A}$. The curves are shown in Figs. 5 and 6 . The curves of the ionizing dose rate, temperature, and induced absorption coefficient of the Suprasil I specimen are very similar to those exhibitex by the Corning 7940 UV specimens. Plateaus of these parameters seem to follow the level of the dose rate employed, with no real decay of the absorption coefficient as the temperature is increased by increased irradiation dose.

The results of the optical transmission of a Suprasil I specimen during irradiation at wavelengths of a $4500 \AA$ are presented in Fig. 6 . It is expected that this curve would be different than those at the previous wavelengths since Corning $7940 \mathrm{UV}$ and Suprasil I fused silica does not have any strong irradiation-induced absorption bands at the 4500 A wavelength. Accordingly, it is no surprise to see at a steady state dose rate with a steady temperature of $400^{\circ} \mathrm{C}$ maintained that the induced absorption coefficient shows a sharp rise and then decays rapidly to a steady lower value untiI the beam is shut off. The curious bump in the induced absorption curve at the end of the run is due to a sudden burst of electrons from the dynamitron during a spark out. The trends, however, for both the fused silica samples are similar. There was some question as to whether heating the specimen by the elctron beam could produce an apparent loss of optical transmission by stresses in the specimen which thereby displace the optical beam off the monochromator slit. In order to test this possibility of a thermal effect erroneously givins a loss in optical transmission, tests were run at $3000 \AA$, a wavelength at which the induced absorption was negligible. Three runs were made using $1.5 \mathrm{~mm}$ samples of Corning $7940 \mathrm{UV}$ fused silica. One test was performed in open air, and one with the sample box evacuated, and one with Helium.

The results clearly show there was no anomal: due to specimen temperature even though very high termperatures were reached by the test sample.

A Corning $7940 \mathrm{UV}$ specimen was soft mounted using a special ceramic material in order to remove any doubt that thermal strains imposed by the sample holder might give erronegus results. The wavelength of the test is $2150 \AA$, and no significant difference in the results are evident using this ceramic soft mounting material. All parameter trends are repeated and verified.

The steady-state irradiation-induced absorption coefficient at $2150 \AA$ over temperatures ranging from $100^{\circ}$ to $1000^{\circ} \mathrm{C}$ are plotted against the ionizing dose rate in Fig. 7. Also shown in Fig. 7 are curves from steady-state irradiations obtained recently from the experiments conducted by Palma and Gagosz in Ref. 8. It is apparent from these curves and data points that there is evidence of disagreement in these two experiments. The claim of Palma and Gagosz that the induced absorption coefficient at a given temperature first rises with increasing dose rate, reaches a maximum value, and then begins to decrease with increasing dose rate is not verified by this experimental work. Although this work has been dore with specimens of the same size, composition and conficuration, the results, as presented by this graph, differ markedly. A thourough examination of their apparatus has failed to reveal to us the cause of this discrepancy.

\section{Discussion and Conclusions}

This experimental study attempted to determine the level of the irradiation-induced absorption expected in the transparent wall of the nuclear light bulb engine (NLB) when the wall is kept at elevated temperatures. The data pertaining to the optical transmission of Corning $7940 \mathrm{UV}$ and Suprasil I fused silica verified the behavior of the induced absorption coefficient as follows:

In irradiations conducted at $2150 \AA$, the wavelength of primary interest, the irradiationinduced absorption coefficient reached a steady state value once the specimen temperature had reached equilibrium.

Increasing the specimen temperature by raising the electron current density or ionizing dose rate caused the induced absorption coefficient to rise to a higher steady state value.

Increasing the temperature of the specimen 
wh le maintaining the same ionizing dose rate resulted in a lower value for the irradiationinduced, absorption coefficient to a lower steady state value.

The irradiation-induced absorption coefficient decreased rapidy after the electron beam was shut down, although sometimes after reaching a minimum value there would be a small increase or hump present caused by the response of the recorder. This investigation provea that Corning 7940 UV and Suprasil $\frac{I}{8}$ at the wavelengths of $2150 \AA, 2700 \AA$ and $4500 \AA$ are approximately the same, with no significant differences between these two materials in effects of temperature or irradiation.

Values ranging from $14.5 \mathrm{~cm}^{-1}$ to $2.2 \mathrm{~cm}^{-1}$ were recorded for the irradiation-induced absorption coefficient at the wavelength of $2150 \AA$ depending upon the test conditions.

At the wavelength of $2700 \AA$ both types of specimens exhibited essentially the same behavior as stated above at $2150 \AA$. The induced absorption coefficient varied between $3 \mathrm{~cm}^{-1}$ to $9.7 \mathrm{~cm}^{-1}$ depending on the test conditions.

The response at the $4500 \AA$ wavelength displayed less transmission loss than at the wavelengths $2700 \AA$ or $21.50 \AA$ as expected. In addition the induced absorption coefficient rose to a maximum value and then decayed rapidly to a lower equilibrium value. This transient change ir transmission was expected since the ultra-pure fused silica used does not exhibit any strong absorption bands at $4500 \AA$.

No anomalous loss of optical transmission was recorded due to the extremely high temperatures experienced by the specimens regardless of whether the specimer environment was air, air-helium gas mixture, or a vacuum. Using a special ceramic gasket material as a soft mount for the fused silica did not alter the results. Measurements of the induced absorption coefficient when the specimen was soft mounted indicated the same trends and almost the identical loss of transmission at the wavelength of $2150 \AA$ as the firm mounted specimen. These two results show no erroneous results due to thermal stresses caused by the original firm mounting of the specimen which had any significant effect on the optical transmission at this important wavelength.

This particular investigation does not give a complete answer as to the practical realization of the nuclear light bulb propulsion scheme. An extension of this study to include the effects of optical bleaching, absorption in the vacuum ultraviolet wavelength region, wavelength scans over the range of $2000 \AA$ to $3000 \AA$, additional test for any anomaly due to high temperature, and the temporal behavior and measurements of the irradiation-induced optical absorption of other promising transparent wall materials such as aluminum oxide, beryllium oxide and titanium oxide should be performed. Since the objective of the present investigation was to measure under simulated reactor environment, at the wavelengths of interest, the optical transmission of fused silica, it is desirable to simulate the actual test conditions as accurately as possible. Therefore, it would be advantageous to extend this work also by utilizing a high energy proton beam which would simulate neutron damage in the specimen. Although there are still areas, as shown above, needing attention in this work, the results of the present investigation indicates significant optical absorption at the wavelengths of interest. This conclusion implies that the feasibility of this concept insofar as it depends upon fused silica transparency under these conditions, is open to question.

\section{Appendix - The Calculation of Electron Dose Rates In Thick Specimens}

Determining the ionizing dose rate due to the penetration of relativistic electrons into specimens which are thick compared to the range of the electron is made more complex because of the inelastic scattering and straggling of the electron energy. The electron traverses along a zig-zag path due to the inelastic scattering. These two effects result in a broad distribution of electron energy loss. If the energy loss is averaged over this broad distribution, the energy in the deposited specimen can be calculated and converted to ionizing dose rate. The useful computational scheme of Burrel, Wright and Watts ${ }^{5}$ which is in part based on the Monte Carlo electron transfer data of Berger 6 is used in this report and by Pa.lma and Gagosz ${ }^{7}$ in their similar work on fused silica. To obtain a sufficient dose rate, it is required that the fraction of the incident electron energy deposited be as large as possible, but it is necessary to trade off this parameter with the fraction of the incident electrons actually stopping in the specimen (keeping this to a minimum so that the charge buildup in the specimen will be small). Burrel, et a ${ }^{5}$ obtain expressions for the fractions above by fitting the data of Berger $^{6}$ and yield the equation below for normal incidence:

$p(l, E)=0.45\left[1-\exp \left(-0.653 z-2.40 z^{2}-6.89 z^{3}\right)\right]$

$n(\ell, \bullet)=0.912\left[1-\exp \left(-0.0512 z+1.128 z^{2}-9.38 z^{3}\right)\right]$

where:

$\mathrm{n}(l, \theta)=$ Fraction of the incident electron energy deposited

$p(\ell, \theta)=$ Fraction of the incident electrons absorbed in the specimen

$=\quad=$ Electron angle of incidence with the specimen surface

also

$$
z=\frac{l}{x_{0}}
$$

where $\mathrm{z}$ represents the fraction of the average path length $x_{O}$ traversed by the electron in penetrating the distance $\ell$. This ratio plotted against the sum of the reflection and transmission factors of Berger show little change with variation in electron energy.

The relationship between the average path length $x_{0}$ and the extrapolated range $R(\vec{F})$ is approximated by: 


$$
\mathrm{z}=\frac{\ell}{(1.33-0.19 \mathrm{E}) \mathrm{R}(\mathrm{E})}
$$

where:

l $=$ specimen thickness $(\mathrm{cm})$

$\mathrm{E}=$ electron kinetic energy (MeV)

$R(E)=$ electron range $(\mathrm{cm})$

With these considerations in mind, the specimen thickness $l$, was chosen as $1.5 \mathrm{~mm}$. This value permits accurate determination of the optical transmission. The range of $1.5 \mathrm{MeV}$ electron in fused silica is $R(E)=4 \mathrm{~mm}$ therefore $z$ is 0.36 , and $p(l, \theta)=0.54$ and $n(l, \theta)=0.25$. Therefore, 25 percent of the penetrating electrons are $a b-$ sorbed giving up 54 percent of their energy in a fused silica sample $1.5 \mathrm{~mm}$ thick. The extreme complexity of the processes involved makes the relation between the ionizing dose rate $\dot{D}$ and the electron density $J$ at a kinetic energy of 1.5 $\mathrm{MeV}$ only an approximation. The relation using the technique described above is the same one used by Palma and Gagosz ${ }^{7}$ and is expressed as:

$$
\begin{aligned}
\dot{\mathrm{D}}=p(l, \theta)\left(\frac{\mathrm{JE}}{\rho l}\right)[1 & +\tan \beta\left(\frac{l}{\omega}\right. \\
& \left.\left.+\frac{1}{3} \frac{l^{2}}{\omega^{2}} \tan \beta\right)\right]^{-1} \times 10^{5} \frac{\mathrm{RAD}}{\mathrm{SEC}}
\end{aligned}
$$

Substituting the values given below into Eq. (5) where

$p(l, \theta)=0.54, \rho$, the density of fused

silica is given as $2.2 \mathrm{gm} / \mathrm{cm}^{2}$ with a specimen thickness $l$ of $0.15 \mathrm{~cm}$ at the electron beam radius $\omega$.

of $0.5 \mathrm{~cm}$ and with $\beta$ the electron beam angle of divergence for fused silica of approximately $60^{\circ}$, Eq. (5) numerically reduces to $D \cong 0.13 \mathrm{~J} \mathrm{M} \mathrm{M}_{\mathrm{RA}} / \mathrm{sec}$ as mentioned previously.

\section{References}

1. Cleland, M. R. and Farrell, P., "Dynamitrons of the Future," IEEE Transactions on Nuclear Science, Vol. 12, No. 3, June 1965, pp. 227234.

2. Edwards, O. J, "Optical Transmittance of Fused Silica at Elevated Temperatures," Journal of the Optical Society of America, Vol. 56, No. 10, Oct. 1966, pp. 1314-1319.

3. Edwards, 0. J., "Optical Absorption Coefficients of Fused Silica in the Wavelength Range 0.17 to 3.5 Microns From Room Temperature to $980^{\circ}$,C, " TN D-3257, 1966, NASA, Cleveland, Ohio.

4. Anon., "Corning Fused Silica Code 7940," Data Sheet, 1964, Corning Glass Works, Corning, N. Y.

5. Burrell, M. 0., Wright, J. J., and Watts, J. W., Jr., "The Calculation of Electron and Bremsstrahlung Dose Rates," Protection Against Space Radiation, SP-169, 1968, NASA, Washington, D.C., pp. 529-538.

6. Berger, M. J., "Transmission and Reflection of Electrons by Aluminum Foils," TN-187, Apr. 1963, National Bureau of Standard, Washington, D.C.

7. Pa.lma, G. E. and Gagosz, R. M., "Optical Absorption in Fused Silica During Irradiation at High-Temperature. H-930709-1, NASA CR107838, Nov. 1969, United Aircraft Corp., East Hartford, Conn.

8. Palma, G. E. and Gagosz, R. M., "Optical Absorption in Transparent Materials During 1.5 MeV Electron Irradiation," J-990929-1, NASA CR-110907, Oct. 1970, United Aircraft Corp., East Hartford, Conn. 


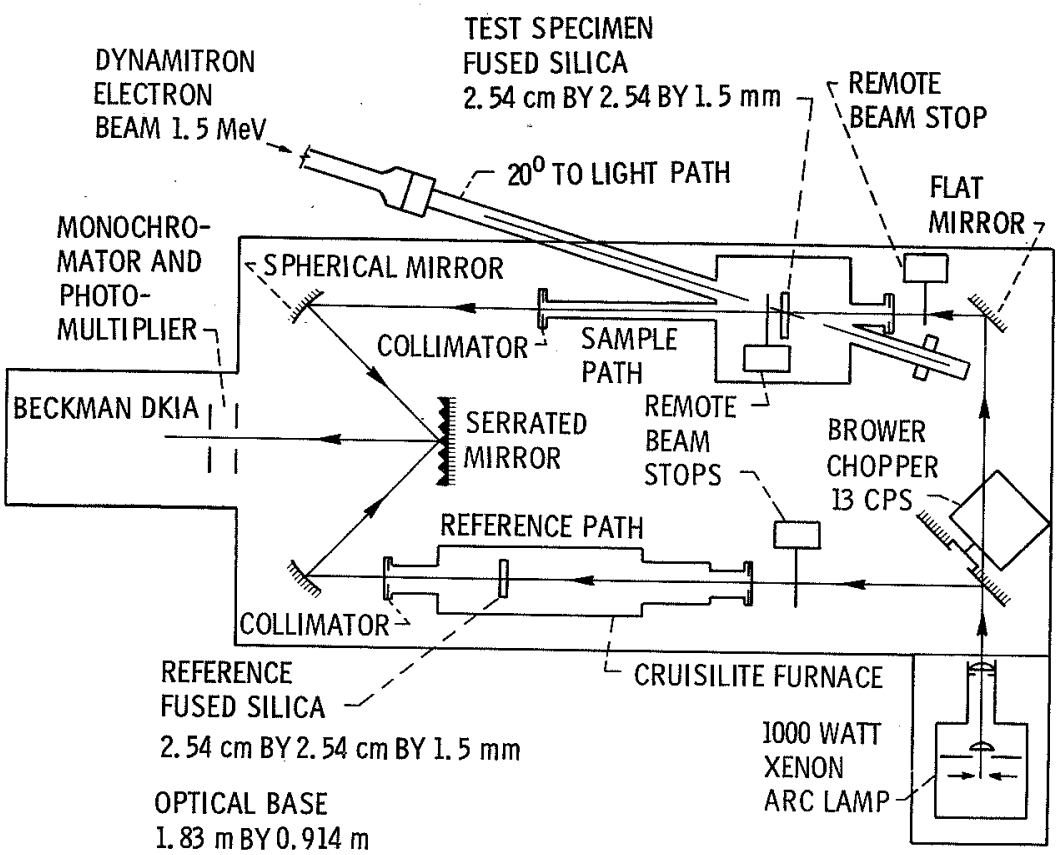

Figure 1. - Diagram of optical reference-sample paths.

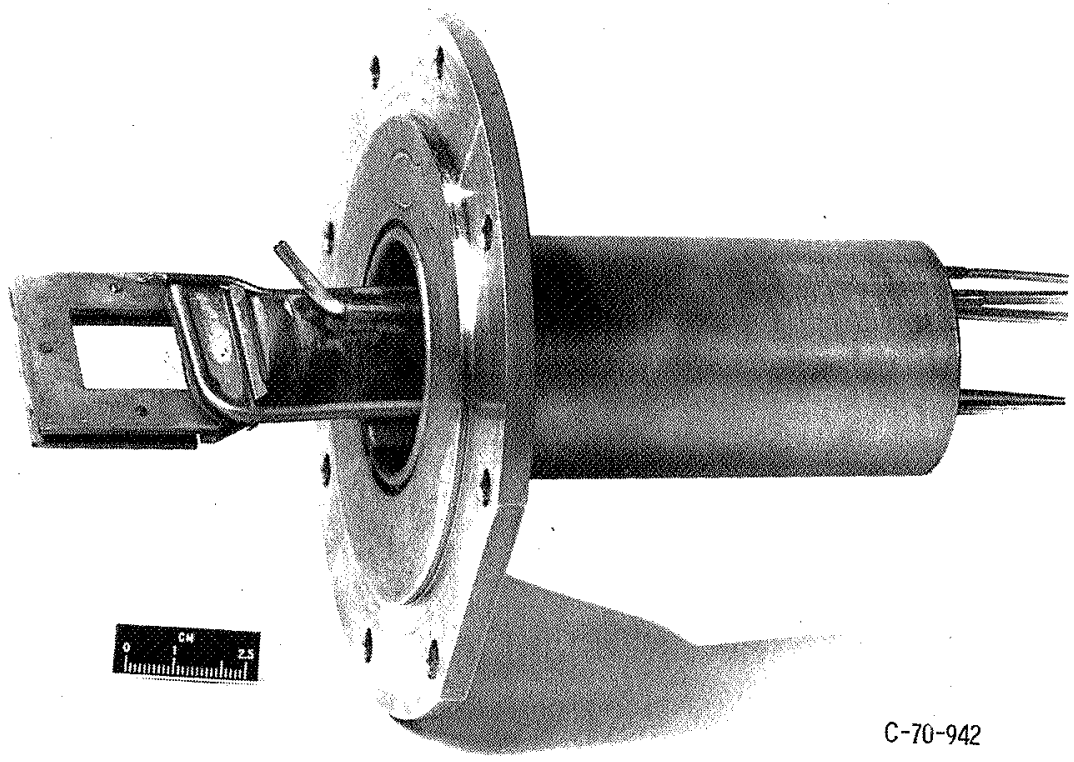

Figure 2. - Specimen holder. 


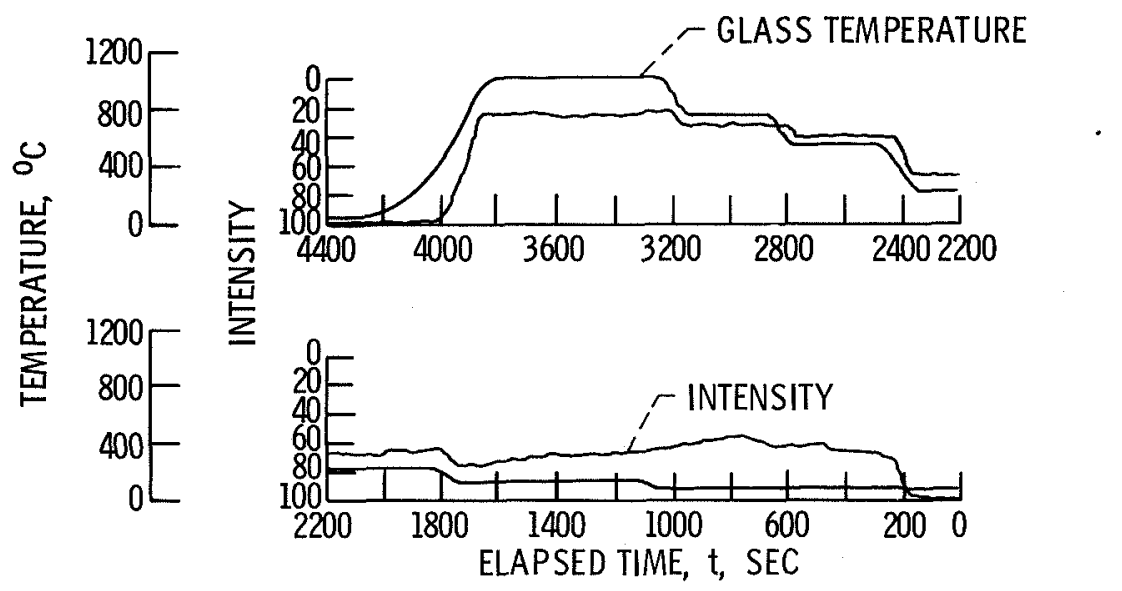

Figure 3. - Typical raw data. (Suprasil I, $1.5 \mathrm{~mm}$ thick specimen at $2700 \AA$ ). 

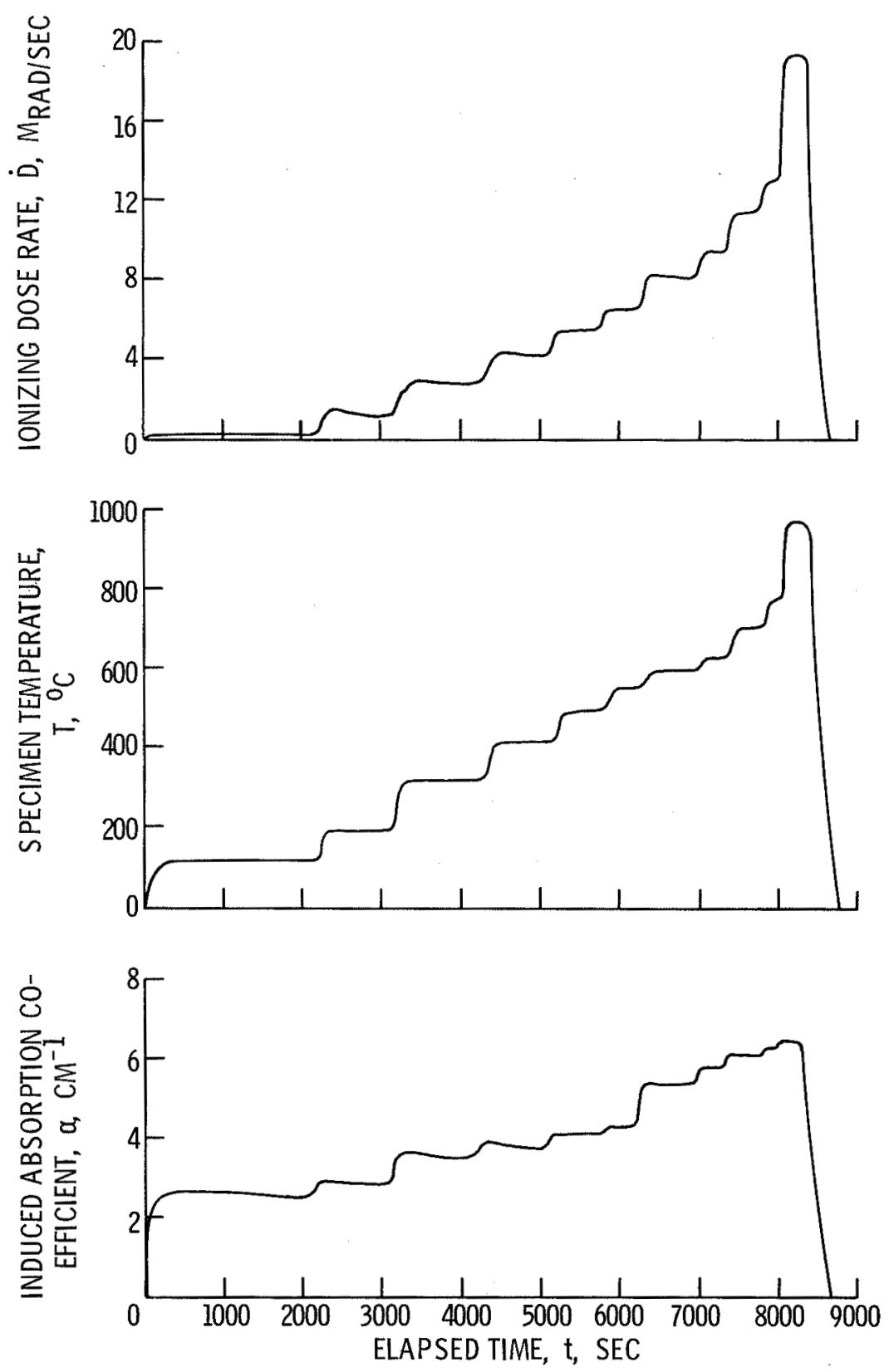

Figure 4. - Electron irradiation history of $1.5 \mathrm{~mm}$ Suprasil I specimen at $2150 \AA$. 

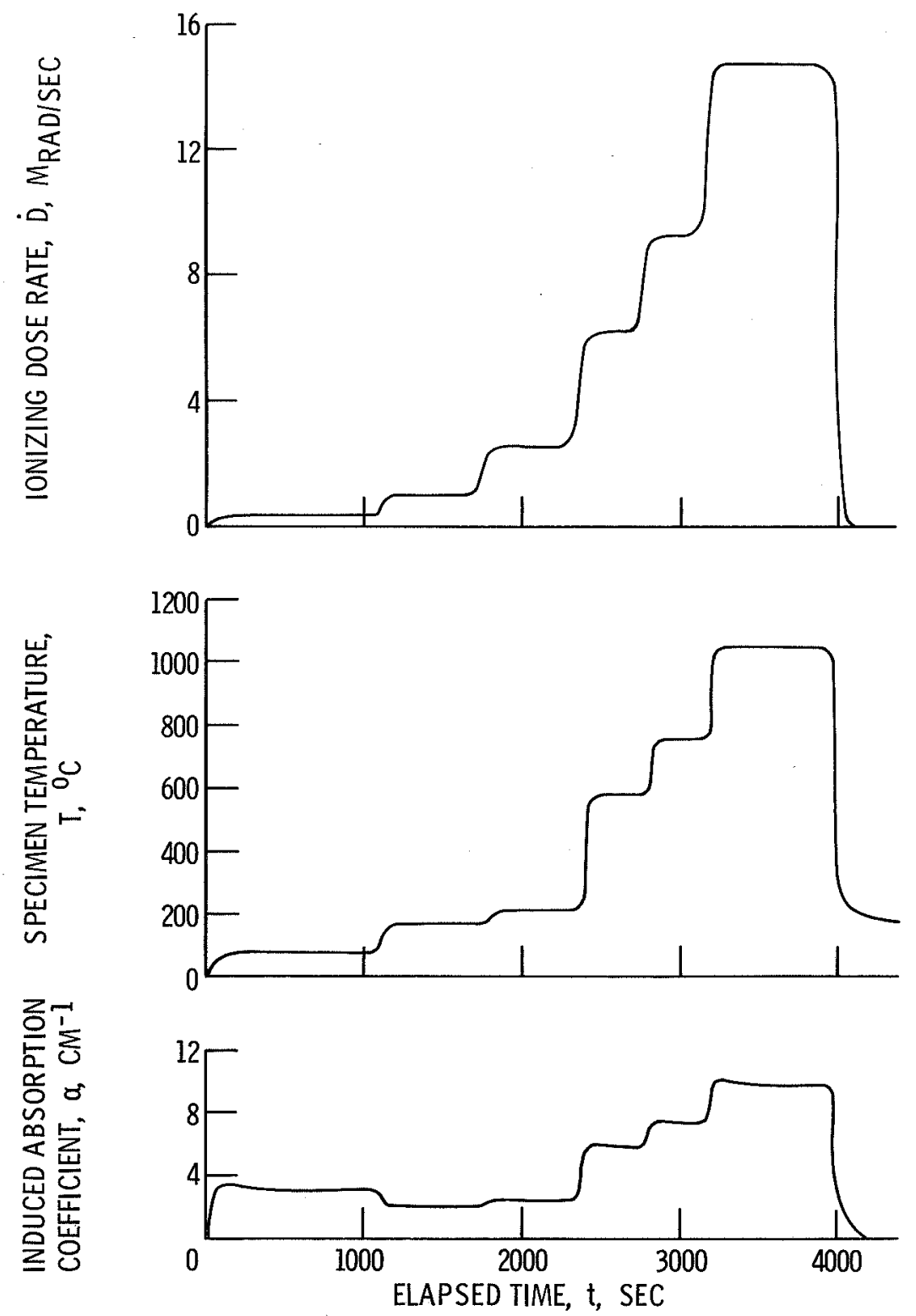

Figure 5. - Electron irradiation history of $1.5 \mathrm{~mm}$, Suprasil I specimen at $2700 \AA$. 

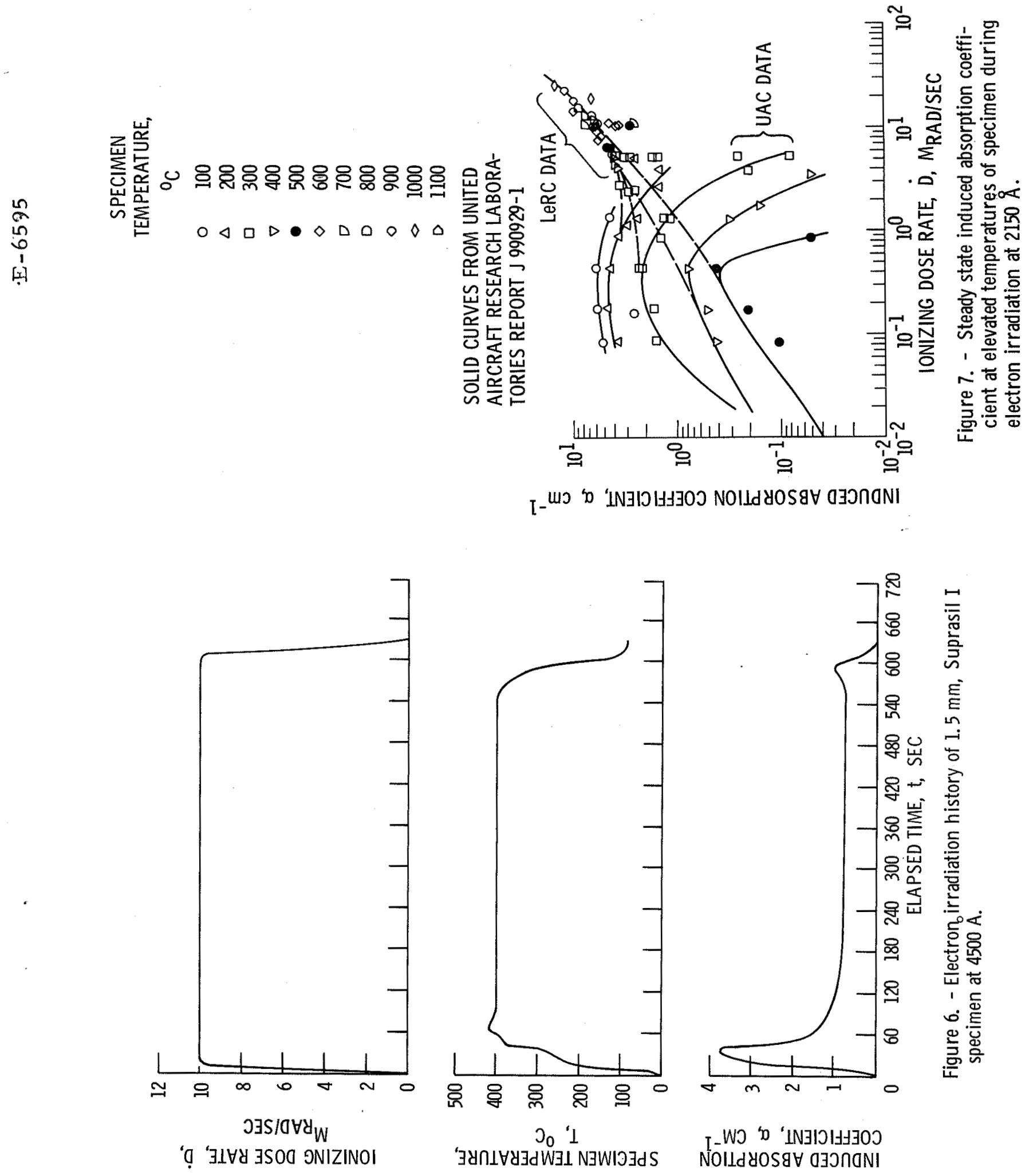